\title{
Characterization of S-adenosylmethionine Synthetase Gene from Red Algae, Pyropia tenera
}

\author{
Jong Won Han', Hyun Dae Hong ${ }^{1}$, Jin-Woo Han', Hyemin Lim², Gang-Seob Lee ${ }^{3}$, Hyun-Ju Hwang * \\ ${ }^{1}$ LMO Technology Development Team, Converging Research Division, National Marine Biodiversity Institute of Korea, Seocheon \\ 33662, Korea \\ ${ }^{2}$ Department of Forest Genetic Resources, National Institute of Forest Science, Suwon 16631, Korea \\ ${ }^{3}$ Department of Agricultural Biotechnology, National Academy of Agricultural Science, Jeonju 55365, Korea
}

\begin{abstract}
S-adenosylmethionine (SAM) is widely involved in variety of biosynthetic processes. It participates in the methionine (Met) metabolic pathway. SAM synthetase (EC 2.5.1.6) is the main enzyme that creates SAM from Met and adenosine triphosphate. $S A M S$ genes are highly conserved in different species. We have isolated SAMS genes from red algae, Pyropia tenera. It has been reported that $S A M S$ genes can confer abiotic stress-related response and growth of plant organs in other species. To elucidate the function of PtSAMS gene in evolutionary perspective, we analyzed SAMS gene using bioinformatics tools and plant transformation of Arabidopsis.
\end{abstract}

Keywords S-adenosylmethionine synthetase, SAMS, Pyropia tenera, Abiotic stress, Organ development

\section{INTRODUCTION}

S-adenosylmethionine synthetase (SAMS), also known as methionine adenosyltransferase (MAT), is an enzyme that synthesizes S-adenosylmethionine (AdoMet or SAM) from methionine and adenosine triphosphate (ATP) (Binet et al. 2011). SAM has been used in many cellular biochemistry reactions. It is the major hub of methionine metabolism (Martínez-López et al. 2008). SAM is a universal methyl donor. It plays a central role in most methylation of DNA, RNA, proteins, lipids, and polysaccharides in numerous enzymecatalyzed reactions (Hilti et al. 2000).

$S A M S$ gene in plant functions in organ development and stress response (Roeder et al. 2009). MAT3 is one of the 4 S-adenosyl methionine synthetase genes in Arabidopsis and is required pollen germination and pollen tube growth
(Chen et al. 2016). PcSAMS1 and PcSAMS2 have been isolated from Pinus contorta (Lindroth et al. 2001). PcSAMS1 is expressed in roots and exhibits a specific expression pattern in the root meristem, whereas PcSAMS2 is expressed in roots as well as in shoots. SAMS have been reported from many plants, including Arabidopsis thaliana (Lindermayr et al. 2005; Mao et al. 2015), Oriza sativa (Van Breusegem et al. 1994), Pinus contorta (Lindroth et al. 2001), and Glycine max (Oh and Komatsu 2015). Differential expression patterns of SAMS have been found in different tissues of Arabidopsis (Shen et al. 2002; Mao et al. 2015). Functional studies have shown the effects of $S A M S$ on abiotic stress. For example, overexpression of $S A M S$ in alfalfa and tobacco could improve their salinity tolerance (Qi et al. 2010; Hua et al. 2012). Low temperature conditions can induce the expression of $S A M S$ in O. sativa, A. thaliana, and Zea mays (Cui et al. 2005;

Received November 18, 2016; Revised November 21, 2016; Accepted November 22, 2016; Published November 30, 2016

*Corresponding author Hyun-Ju Hwang, hjhwang@mabik.re.kr, Tel: +82-41-950-0761, Fax: +82-82-41-950-0765 
Yang et al. 2006; Uváčková et al. 2012). Consequently, it is important to determine the functions of SAMS in plant development and abiotic stress related mechanism. Stress-related gene SAMS (PySAMS) has been cloned from seaweed, Porphyra yezoensis (Yi et al. 2009). However, the exact functions of SAMS remain unknown. Therefore, the objective of this study was to perform functional analysis for PtSAMS through in silico study and generation of PtSAMS transgenic plant.

\section{MATERIALS AND METHODS}

\section{Plant material and growth condition}

Wild type seeds were surface sterilized in $70 \%$ ethanol containing 3.5\% (w/v) household bleach and $0.1 \%(\mathrm{w} / \mathrm{v})$ Tween 20 detergent for $20 \mathrm{~min}$ and subsequently rinsed by $100 \%$ ethanol 5 times (Kalifa et al. 2004). After entirely dried, sterilized seeds were carefully scattered onto $0.5 \times$ Murashige and Skoog (MS) plate containing $0.8 \%$ agar and $2 \%$ sucrose. To improve the rate and synchrony of germination, plates were placed at $4^{\circ} \mathrm{C}$ for 3 days (Li et al. 2013). Arabidopsis were then grown at $22^{\circ} \mathrm{C}$ with light intensity of $120 \mu \mathrm{mol}$ photons $\mathrm{m}^{-2} \mathrm{sec}^{-1}, 16 / 8$ (light/dark) photoperiod, and relative humidity of $60 \%$. After germination for 10 days, Arabidopsis seedlings were then transferred to soil. Arabidopsis were allowed to grow until bolting occurred on soil.

\section{Construction of 35S::PtSAMS expression vector}

The full-length cDNA of PtSAMS gene was amplified from Pyropia tenera cDNA library using PCR from Pyropia yezoensis sequence (GenBank: FJ404748.1). The following primers were used: PySAMS-OX-XbaI-F (5'AATCTAGAATGGCAGCCATGAAGAAC-3') and PySAMS-OX-SmaI-R (5'-AACCCGGGCTAGAGCTCA AGC-3'). Underlines are restriction enzyme sites for XbaI and SmaI, respectively.

\section{Generation of transgenic plants}

To generated transgenic plants, Arabidopsis were transformed using GV3101 strain of Agrobacterium tumefaciens according to the floral dip protocol (Clough and Bent 1998). Transformed seeds (T0) were selected on $0.5 \times \mathrm{MS}$ containing $4 \mathrm{mg} / \mathrm{L}$ phosphinothricin $(\mathrm{PPT})$ and $250 \mathrm{mg} / \mathrm{L}$ cefotaxime.

\section{Genomic DNA extraction and genotyping}

Genomic DNAs of transgenic plants were extracted using GeneAll Plant SV kit (GeneAll, Seoul, Korea). bar gene selection of transgenic plants was carried out using PCR with the following primers: bar-F (5'-GGATCTACCATGAGCCCAGA-3') and bar-R (5'-GGAAGTTGACCGTG CTTGTC-3'). PCR conditions included an initial denaturation for $94^{\circ} \mathrm{C}$ for 5 minutes followed by 30 cycles of denaturation for 30 seconds at $94^{\circ} \mathrm{C}$, annealing for 30 seconds at $55^{\circ} \mathrm{C}$, extension for 30 seconds at $72^{\circ} \mathrm{C}$, and 7 minutes of final extension at $72^{\circ} \mathrm{C}$. Emerald Amp GT PCR master mix (TaKaRa, Shiga, Japan) were used for PCR reactions.

\section{Sequence analysis}

Protein domain and family search were performed using ExPASy Bioinformatics Resource Portal (http:// www.expasy.org). PtSAMS and protein sequences of other SAM synthetases of different species including bacterial, fungi, plants, and animals were searched from National Center for Biotechnology Information sequence database (DB) and aligned using Geneious software ver 9.1.6. Phylogenetic tree of PtSAMS protein was constructed by Geneious software ver. 9.1.6. using a neighbor-joining (NJ) method with bootstrap set at 1,000 replicates (Saitou and Nei 1987).

\section{RNA extraction and quantitative real-time PCR}

Total RNA was isolated using Hybrid-R RNA purification kit (GeneAll, Seoul, Korea) according to the manufacturer's instruction Two micrograms of total RNAs were used for cDNA synthesis using amfiRivert Platinum cDNA synthesis master mix (GenDEPOT, Barker, TX, USA). Quantitative real-time PCR (qRT-PCR) was carried out on Dice Real Time System Single Thermal Cycler (TaKaRa) using SYBR ${ }^{\circledR}$ Premix Ex Taq $^{\text {TM }}$ (TaKaRa). PtSAMS gene expression levels in transgenic plants were determined by qRT-PCR using the following primers: PtSAMS-F (5'-GAGGA CCTGATGCCCTTGAC-3') and 
PtSAMS-R (5'-CTGCGTGCTGATGACAATCG-3'). AtActin2 gene was used as internal control. AtActin2-F (5'GATGAGGCAGGTCCAGGAATC-3') and AtActin2-R (5'-AACCCCAGCTTTTTAAGCCTTT-3') were used for qRT-PCR with the following parameters: $94^{\circ} \mathrm{C}$ for 30 seconds followed by 40 cycles at $94^{\circ} \mathrm{C}$ for 5 seconds and $60^{\circ} \mathrm{C}$ for 30 seconds. Relative expression level was calculated using the $2^{-\Delta \Delta \mathrm{CT}}$ method (Livak and Schmittgen 2001).

\section{Abiotic stress assay}

For Arabidopsis seedling assays, wild type and PtSAMS overexpressing transgenic lines seeds were surface sterilized and germinated on $0.5 \times \mathrm{MS}$ medium supple-

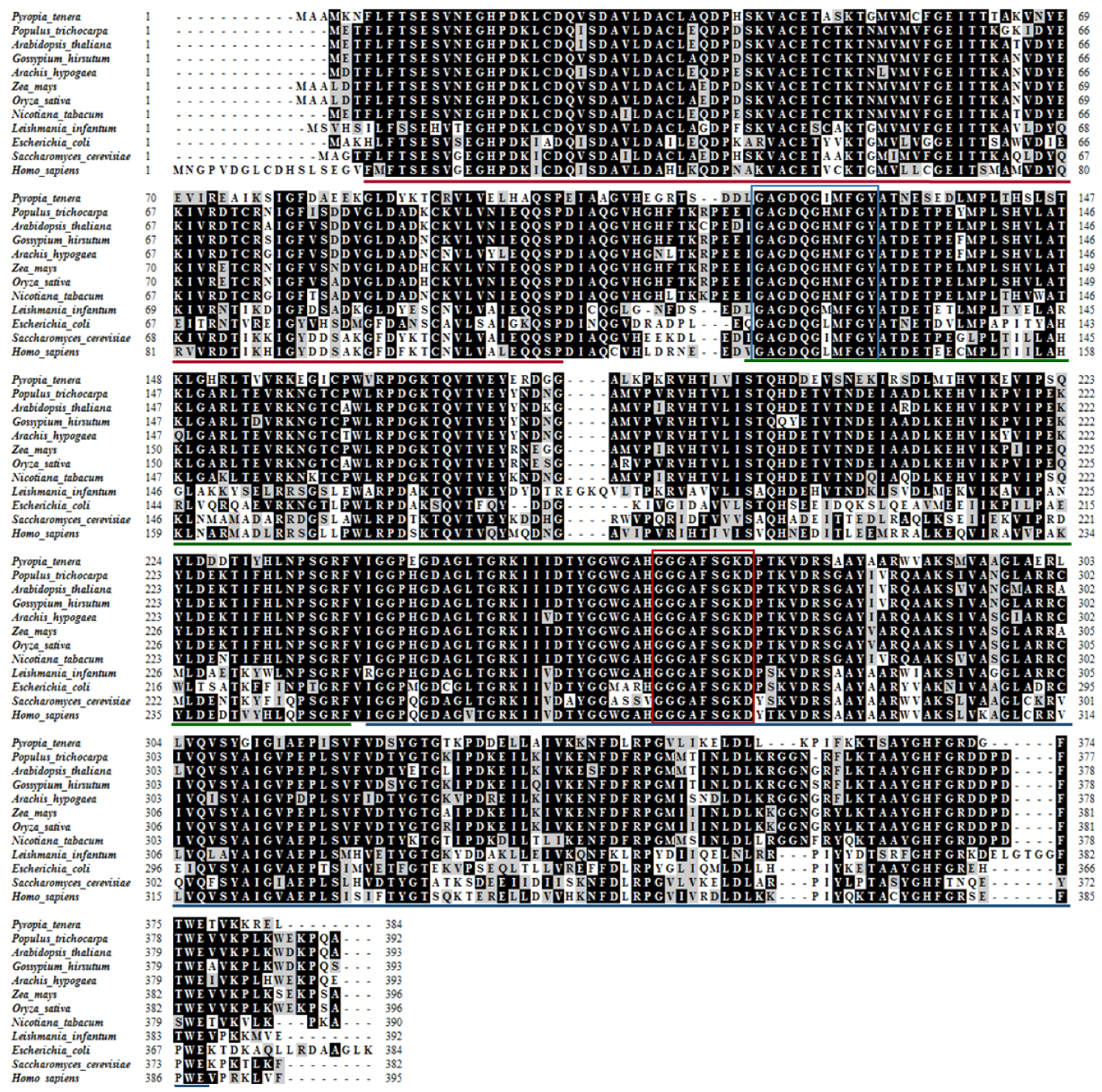

Fig. 1. Multiple alignments of S-adenosylmethionine synthetase (SAMS). The deduced amino acid sequence of SAMS of Pyropia tenera were aligned with Populus trichocarpa (EEE99264.1), Arabidopsis thaliana (AAA32868.1), Gossypium hirsutum (ADN96174.1), Arachis hypogaea (AFN54653.1), Zea mays (ACG42196.1), Oryza sativa (CAA81481.1), Nicotiana tabacum (NP_001312332.1), Leishmania infantum (AAB88448.2), Escherichia coli str. K-12 substr. MG1655(NP_417417.1), Saccharomyces cerevisiae (P10659.2) and Homo sapiens (NP_000420.1). The boxes indicate the binding sites. Blue box: S-adenosylmethionine synthase signature motif (PS00376), [GN]-[AS]G-D-Q-G-x(3)-G-[FYHG] and red box: S-adenosylmethionine synthase signature 2 (PS00377), G-[GA]-G-[ASC][FY]-S-X-K-[DE]. The under lines indicate the conserved domains. Red underline: S-adenosylmethionine synthetase N-terminal domain (PF00438), green underline: S-adenosylmethionine synthetase central domain (PF02772), and blue underline: S-adenosylmethionine synthetase C-terminal domain (PF02773). 
mented with different concentrations, $0 \mathrm{mM}, 50 \mathrm{mM}, 100$ $\mathrm{mM}$, or $200 \mathrm{Mm}$ of $\mathrm{NaCl}$ and $\mathrm{KCl}$, respectively as plant growth condition methods. Seedlings that were completely bleached were considered dead and those with green leaves are considered surviving at 12-day-old plants (Divi et al. 2010). To evaluate the stress tolerance of transgenic lines, plants were vertically grown on the agar plates. Root lengths were compared wild type and PtSAMS-OX. Seedlings were photographed and root lengths were measured (Chen et al. 2014). These experiments were repeated three times.

\section{RESULTS}

\section{Amino acid sequence analysis and alignment}

We isolated the $S A M S$ gene from red algae, P. tenera. $S A M S$ genes are conserved in various species such as bacteria, fungi, plants, and animals. The function of SAMS has been reported to confer plant organ development.
MAT3 is one of the $4 \mathrm{~S}$-adenosyl methionine synthetase genes in Arabidopsis and is required pollen germination and pollen tube growth (Lindroth et al. 2001; Chen et al. 2016). PCSAMS1 and PCSAMS2 have been isolated from Pinus contorta. PcSAMS1 is specific to root growth and formation, while PcSAMS2 functions more generally during plant growth (Lindroth et al. 2001). SAMS from Panax ginseng was significantly under salt, salicylic acid, abscisic acid and chilling stresses (Pulla et al. 2009). PySAMS gene isolated from Porphyra yezoensis was known to be stress related gene. However, its function in plants is unknown.

To better understand the function of PtSAMS, we performed sequence alignment using deduced amino acid sequences of SAMS from P. tenera, E. coli, Human, and other species. SAMS are highly conserved among all species tested. Three main domains are highly conserved (Fig. 1). Previous study has reported that two SAMS signature motifs, GAGDQGHMFGY and GGGAFSGKD, are conserved among many species (Hilti et al. 2000; Qi et

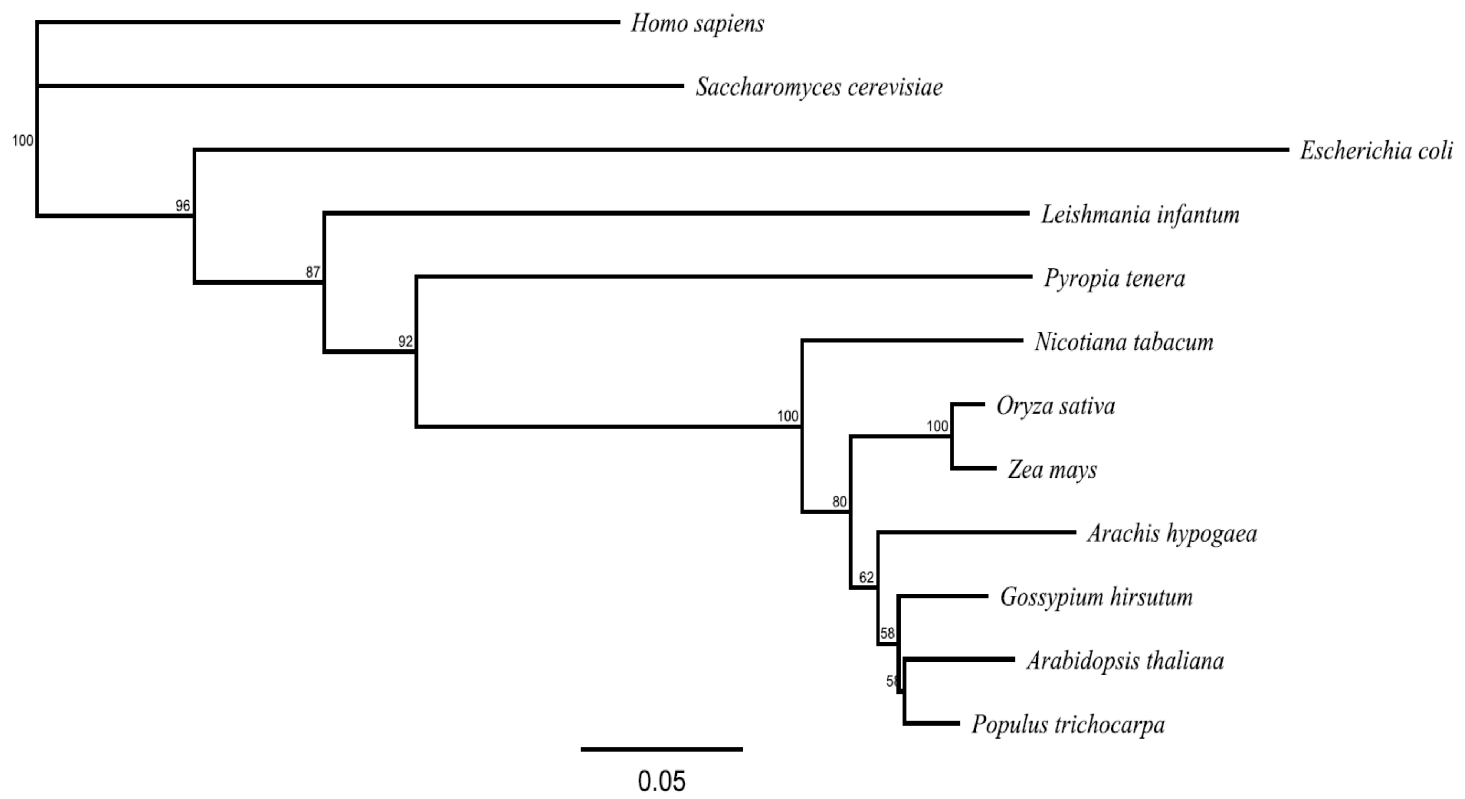

Fig. 2. Amino acid sequence-based phylogenetic analysis of PtSAMS and other species SAMS proteins. Phylogenetic tree was constructed using the Geneious software ver. 9.1.6 with Neighbor-Joining methods. Numbers indicate posterior probabilities values and probability threshold was selected as over 50\%. The sequence used for generating phylogenetic tree are Populus trichocarpa (EEE99264.1), Arabidopsis thaliana (AAA32868.1), Gossypium hirsutum (ADN96174.1), Arachis hypogaea (AFN54653.1), Zea mays (ACG42196.1), Oryza sativa (CAA81481.1), Nicotiana tabacum (NP_001312332.1), Leishmania infantum (AAB88448.2), Escherichia coli str. K-12 substr. MG1655(NP_417417.1), Saccharomyces cerevisiae (P10659.2) and Homo sapiens (NP_000420.1). 
al. 2010). PtSAMS shares three conserved amino acid sequences with other species. These sequences include the conserved methionine binding motif 17-GHPDK-21, the active signature for ATP binding site 120-GAGDQG-125 which is known as the consensus sequence from ATP binding, and the conserved peptide (Li et al. 2013). SAMS sequences share two SAMS signature motifs: the active signature for ATP binding site 120-GAGDQGIMFGY-130 which is known as the consensus sequence for ATP binding and the conserved peptide 267-GGGAFSGKD-275 which forms a P-loop for the phosphate-binding region.

Phylogenetic analysis of SAMS was performed with Geneious software ver 9.1.6 using NJ method. Results are shown in Fig. 2. Based on SWISS-MODEL spatial structure

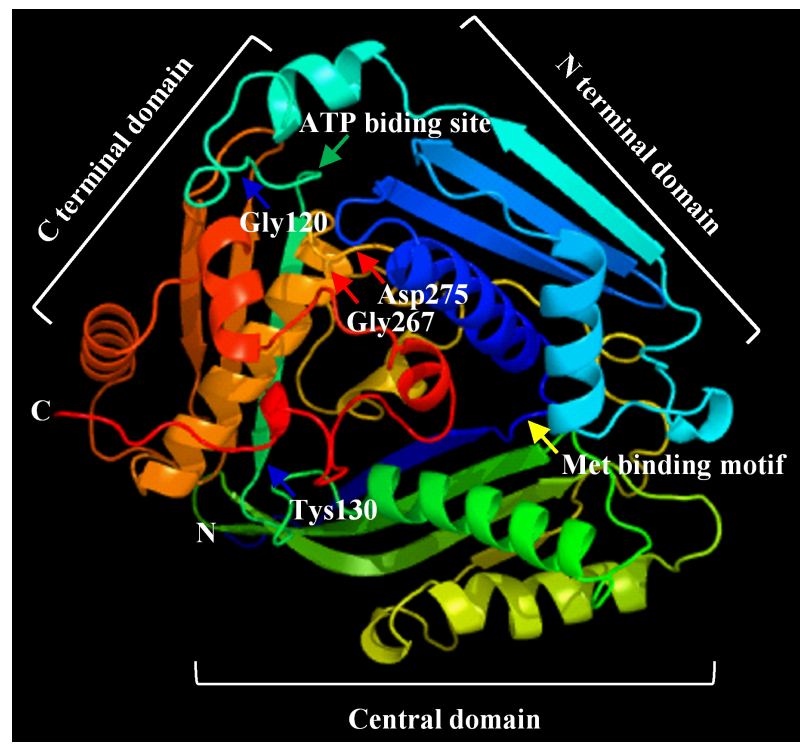

Fig. 3. Analysis of three-dimensional structure of the PtSAMS protein by Phyre $^{2}$ programs. The 3-dimensional model of PtSAMS protein was generated on the basis of the protein data bank (PDB) template of Homo sapiens S-adenosylmethionine synthetase protein (PBD ID: c2obvA). Blue arrow (S-adenosylmethionine synthase signature modif; PS00376), 120GAGDQGIMFGY-130 and red arrow (Sadenosylmethionine synthase signature modif 2; PS00377), 267- GGGAFSGKD-275. Yellow arrow (methionine binding motif): 17- GHPDK-21 and green arrow (ATP biding site): 120-GAGDQG-125. The amino and carboxylic terminal are labeled $\mathrm{N}$ and $\mathrm{C}$, respectively. Each SAM synthetase domains are marked with white line.

ATP: adenosine triphosphate. analysis, the enzyme monomer had three domains: the $\mathrm{N}$-terminal domain, the central domain, and the C-terminal domain (Fig. 3). In addition, PtSAMS was predicted to have two substrate binding sites: a site for ATP binding between the central and C-terminal domains and a Met binding site between the central and $\mathrm{N}$-terminal domain (Fig. 3) (González et al. 2000).

\section{Production and analysis of 35S::PtSAMS transgenic plants}

To elucidate the function of PtSAMS in plants, PtSAMS gene was isolated from cDNA library of $P$. tenera using RT-PCR and introduced into a pPZP vector consisting of 35S CaMV promoter, PinII terminator, and bar genes (Fig. 4A). We generated T0 pool by floral dipping method in Arabidopsis. To select transgenic plant, T0 seeds were grown on PPT containing $0.5 \times \mathrm{MS}$ media. After extraction of genomic DNAs from selected T1 transgenic plants, PCR

A

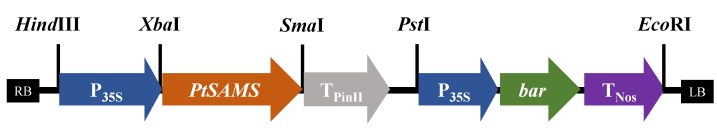

B

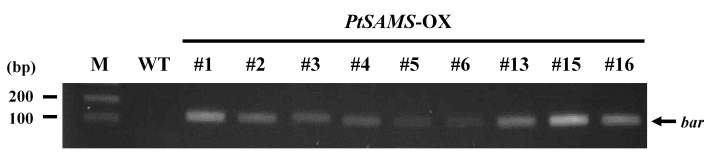

C

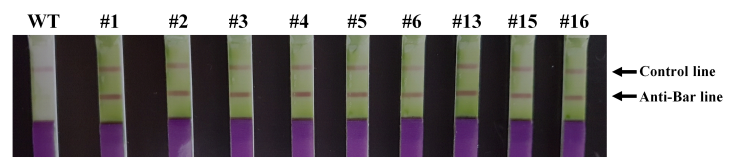

Fig. 4. Schematic representation of the expression vector constructs for PtSAMS overexpression lines. (A) Constructs used for the overexpression of PtSAMS, for which each full length cDNA was cloned into the plant expression vector that harbored a phosphinothricin (PPT) resistance gene. (B) Polymerase chain reaction analysis of $35 \mathrm{~S}::$ PtSAMS transgenic T1 plants by the bar gene specific primer. M: marker, WT: wild type (Ler), lane 3-11: transgenic plants (T1). (C) Detection of bar gene expression of $35 S: \because P t S A M S$ transgenic plants using anti-Bar immune strip.

RB: right border, LB: left border, OX: overexpression, M: marker, WT: wild type (Ler). 
analysis and Bar immune strip test were performed to select transgenic plants that possessed the bar gene (Fig. 4B, C). We have obtained 9 transgenic lines. Afterward, the selected two $\mathrm{T} 1$ plants were used to produce the $\mathrm{T} 2$ generation to separate homozygous lines for further analysis.

\section{Expression of PtSAMS gene in transgenic plants}

To verify the transcriptional levels of PtSAMS gene, transgenic lines were subjected to qRT-PCR analysis. 35S::PtSAMS \#4 and \#16 showed higher levels of expression compared to wild type (Fig. 5). 35S::PtSAMS\#3, $\# 5$, \#6, and \#15 showed relative lower levels of expression compared to the two transgenic lines \#4 and \#16. Therefore, these two lines were used in abiotic stress assays.

\section{Analysis of abiotic stress tolerance}

To investigate the effect of PtSAMS on Arabidopsis under abiotic stress, seeds of wild type and transgenic lines were germinated on $0.5 \times \mathrm{MS}$ medium supplemented with 0 $\mathrm{mM}$ (normal condition), $50 \mathrm{mM}, 100 \mathrm{mM}$, or $200 \mathrm{mM}$ of

A

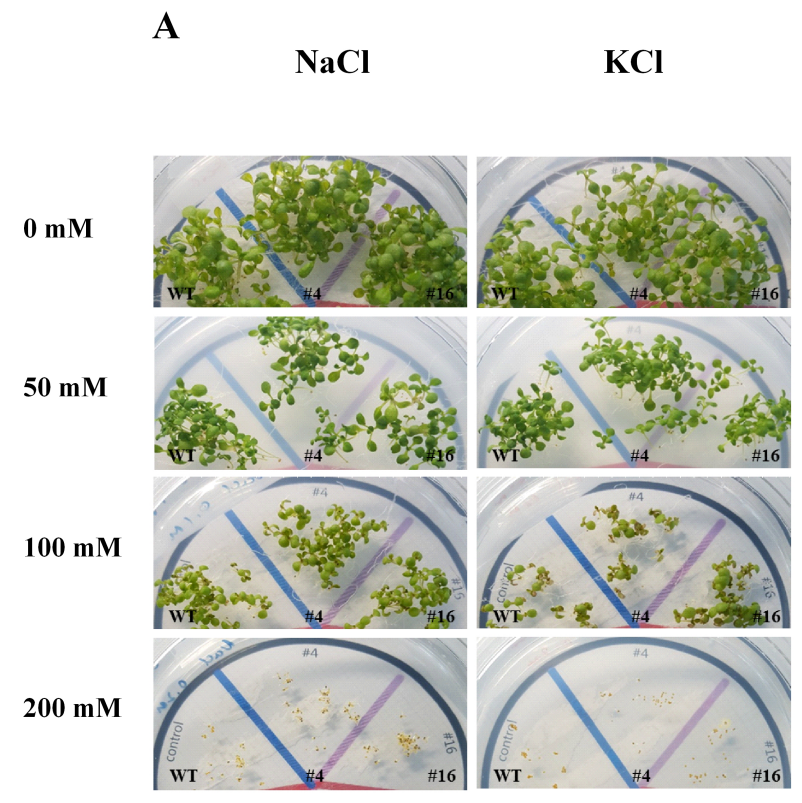

$\mathrm{NaCl}$ and $\mathrm{KCl}$. Growth patterns of both wild type and transgenic lines were observed at 12-day-old plants and root length were measured (Fig. 6). The experimental

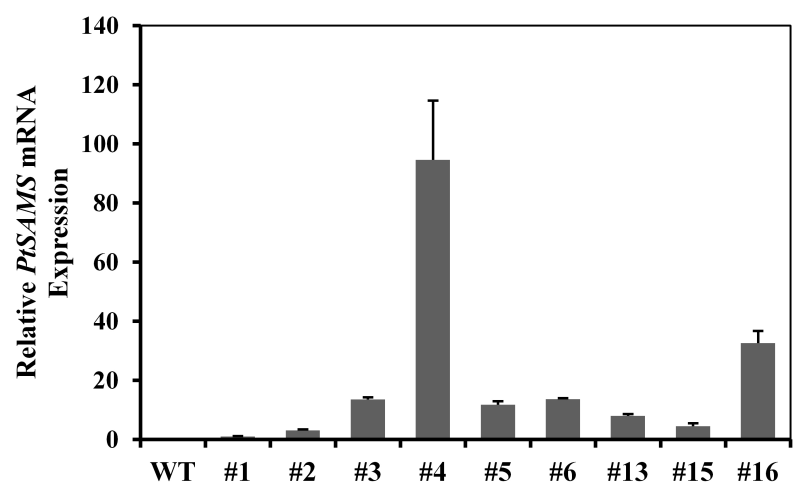

Fig. 5. Analysis of the transgene expression in the transgenic plants. Expression levels of the PtSAMS gene in transformants. AtActin2 gene was used as internal control. Quantitative real-time polymerase chain reaction was analyzed via the $2^{-\Delta \Delta \mathrm{CT}}$ method. The results are presented as average values with standard deviation using each three times.

WT: wild type (Ler).

\section{B}

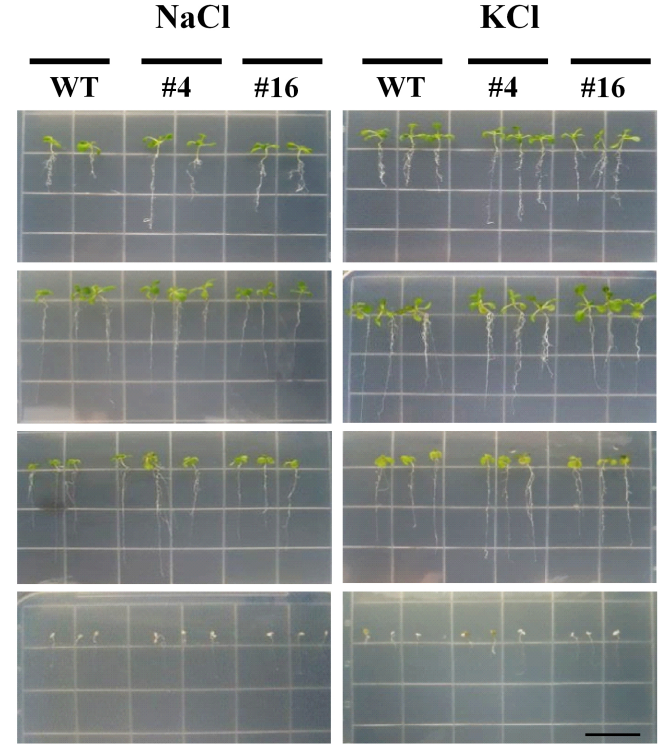

Fig. 6. Comparison of the growth of wild type and transgenic lines overexpressing the PtSAMS gene under salinity stress. (A) The growth was assayed on medium containing $0 \mathrm{mM}, 50 \mathrm{mM}, 100 \mathrm{mM}$, or $200 \mathrm{mM} \mathrm{NaCl}$ and $\mathrm{KCl}$ from the top. The growth pattern was analyzed within 12 days after sowing in medium containing each concentration of $\mathrm{NaCl}$ and $\mathrm{KCl}$. (B) Root length was measured 12 days after seeding. This experiment was repeated three times. Bar: $2 \mathrm{~cm}$.

WT: wild type (Ler). 
results revealed that there was no significant difference in growth patterns and root length from 12-day-old plants between transgenic lines and wild type under abiotic stress conditions (Fig. 6).

\section{DISCUSSION}

SAM is an important molecule that plays a central role in many cellular biological reactions and methionine metabolism (Martínez-López et al. 2008). Biosynthesis of SAM is catalyzed by SAMS using methionine and ATP (Shen et al. 2002). SAMS is also highly conserved throughout the eukaryotic and prokaryotic kingdoms. PtSAMS consists of three domains for S-adenosylmethionine synthesis. It has two substrate binding sites with representative features (Fig. 1,2).

Previous studies have shown that SAMS genes have differential expression patterns in Arabidopsis such as roots, stems, pea ovaries, and rice leaves. In addition, $S A M S$ gene can influence adventitious root development in black locust. MAT3, an S-adenisylmethionone synthetase expressed in pollen, plays an important role during pollen germination and pollen tube growth (Chen et al. 2016).

SAMS catalyzes the synthesis of SAM, a precursor of ethylene, and polyamine (PA) biosynthesis (Wawrzynska et al. 2015). Ethylene mainly regulates germination, adventitious root formation, growth, development, and senescence in plants (Roy et al. 1972; Ge et al. 2006). PAs are ubiquitously present in nature. They are required for cell growth (Fujita et al. 2012). Increase in endogenous amounts of PAs can be induced by environmental stresses including osmotic pressure, temperature, and $\mathrm{pH}$ (Pang et al. 2007). Furthermore, oxidative stress can induce PA biosynthesis and PA content including spermidin and spermine (Smirnova et al. 2012). Some reports have revealed that overexpression of SAMS gene can enhance the tolerance of tobacco against abiotic stress and increase the PA content in tobacco (Luo et al. 2016).

Overexpression of Medicago SAMS gene can also enhance salt tolerance and strength roots (Hua et al. 2012). Besides, upregulation of Chorispora bungeana SAMS involves the content of PAs to deal with cold and high salinity stress (Ding et al. 2015).

To examine the function of PtSAMS in plants, we generated transgenic plants of Arabidopsis (Fig. 4). Salinity stress assay was carried out in wild type and selected transgenic lines plants after treatment with different concentrations of $\mathrm{NaCl}$ and $\mathrm{KCl}$. However, there was no significant difference in germination rate or growth pattern between wild type and PtSAMS-OX transgenic line (Fig. 6). To investigate the role of PtSAMS under salinity stress tolerance, germination rate was observed at 12-day-old plants after $0 \mathrm{mM}, 50 \mathrm{mM}, 100 \mathrm{mM}$, or $200 \mathrm{mM}$ $\mathrm{NaCl}$ and $\mathrm{KCl}$ treatment, respectively. No difference was observed between wild type and transgenic plant lines (\#4 and \#16) in the growth patterns and root length under salinity stress treatment. One of the reasons suggests that PtSAMS gene does not function in plants because of difference of sequence similarity between Arabidopsis and red algae. Another possibility is that $S A M S$ gene may play a role the organ development like OsSAMSs and AtSAMSs in plants (Lucena et al. 2015). Therefore, to better understand the function of PtSAMS gene, further studies are needed to analyze plant development and growth.

\section{ACKNOWLEDGEMENTS}

This study was supported by a grant (2016M00500) funded by National Marine Biodiversity Institute of Korea Research Program. It was also supported by a grant (Project No. PJ0118292016) from the Next-Generation Biogreen 21 Program funded by Rural Development Administration, Jeonju, Republic of Korea.

\section{REFERENCES}

Binet R, Fernandez RE, Fisher DJ, Maurelli AT. 2011. Identification and characterization of the Chlamydia trachomatis L2 S-adenosylmethionine transporter. MBio 2: e00051-00011.

Chen X, Huang Q, Zhang F, Wang B, Wang J, Zheng J. 2014. ZmCIPK21, a maize CBL-interacting kinase, enhances salt stress tolerance in Arabidopsis thaliana. Int. J. Mol. 
Sci. 15: 14819.

Chen Y, Zou T, McCormick S. 2016. S-adenosylmethionine synthetase 3 is important for pollen tube growth. Plant Physiol. 172: 244-253.

Clough SJ, Bent AF. 1998. Floral dip: A simplified method for Agrobacterium-mediated transformation of Arabidopsis thaliana. Plant J. 16: 735-743.

Cui S, Huang F, Wang J, Ma X, Cheng Y, Liu J. 2005. A proteomic analysis of cold stress responses in rice seedlings. Proteomics 5: 3162-3172.

Ding C, Chen T, Yang Y, Liu S, Yan K, Yue X, et al. 2015. Molecular cloning and characterization of an S-adenosylmethionine synthetase gene from Chorispora bungeana. Gene 572: 205-213.

Divi UK, Rahman T, Krishna P. 2010. Brassinosteroidmediated stress tolerance in Arabidopsis shows interactions with abscisic acid, ethylene and salicylic acid pathways. BMC Plant Biol. 10: 151.

Fujita M, Fujita Y, Iuchi S, Yamada K, Kobayashi Y, Urano $\mathrm{K}$, et al. 2012. Natural variation in a polyamine transporter determines paraquat tolerance in Arabidopsis. Proc. Natl. Acad. Sci. U.S.A. 109: 6343-6347.

Ge C, Cui X, Wang Y, Hu Y, Fu Z, Zhang D, et al. 2006. BUD2, encoding an S-adenosylmethionine decarboxylase, is required for Arabidopsis growth and development. Cell Res. 16: 446-456.

González B, Pajares MA, Hermoso JA, Alvarez L, Garrido F, Sufrin JR, et al. 2000. The crystal structure of tetrameric methionine adenosyltransferase from rat liver reveals the methionine-binding site. J. Mol. Biol. 300: 363-375.

Hilti N, Gräub R, Jörg M, Arnold P, Schweingruber AM, Schweingruber ME. 2000. Gene sam1 encoding adenosylmethionine synthetase: Effects of its expression in the fission yeast Schizosaccharomyces pombe. Yeast 16: $1-10$.

Hua Y, Zhang BX, Cai H, Li Y, Bai X, Ji W, et al. 2012. Stress-inducible expression of GsSAMS2 enhances salt tolerance in transgenic Medicago sativa. Afr. J. Biotechnol. 11: 4030-4038.

Kalifa Y, Perlson E, Gilad A, Konrad Z, Scolnik PA, Bar-Zvi D. 2004. Over-expression of the water and salt stress-regulated Asr1 gene confers an increased salt tolerance. Plant Cell Environ. 27: 1459-1468.

Li XD, Xia B, Wang R, Xu S, Jiang YM, Yu FB, et al. 2013. Molecular cloning and characterization of S-adenosyl- methionine synthetase gene from Lycoris radiata. Mol. Biol. Rep. 40: 1255-1263.

Lindermayr C, Saalbach G, Durner J. 2005. Proteomic identification of S-nitrosylated proteins in Arabidopsis. Plant Physiol. 137: 921-930.

Lindroth AM, Saarikoski P, Flygh G, Clapham D, Grönroos $\mathrm{R}$, Thelander M, et al. 2001. Two S-adenosylmethionine synthetase-encoding genes differentially expressed during adventitious root development in Pinus contorta. Plant Mol. Biol. 46: 335-346.

Livak KJ, Schmittgen TD. 2001. Analysis of relative gene expression data using real-time quantitative $\mathrm{PCR}$ and the 2(-Delta Delta C(T)) method. Methods 25: 402-408.

Lucena C, Romera FJ, García MJ, Alcántara E, Pérez-Vicente R. 2015. Ethylene participates in the regulation of $\mathrm{Fe}$ deficiency responses in strategy I plants and in rice. Front. Plant Sci. 6: 1056.

Luo P, Shen Y, Jin S, Huang S, Cheng X, Wang Z, et al. 2016. Overexpression of Rosa rugosa anthocyanidin reductase enhances tobacco tolerance to abiotic stress through increased ROS scavenging and modulation of ABA signaling. Plant Sci. 245: 35-49.

Mao D, Yu F, Li J, Van de Poel B, Tan D, Li J, et al. 2015. FERONIA receptor kinase interacts with S-adenosylmethionine synthetase and suppresses S-adenosylmethionine production and ethylene biosynthesis in Arabidopsis. Plant Cell Environ. 38: 2566-2574.

Martínez-López N, Varela-Rey M, Ariz U, Embade N, Vazquez-Chantada M, Fernandez-Ramos D, et al. 2008. S-adenosylmethionine and proliferation: New pathways, new targets. Biochem. Soc. Trans. 36: 848-852.

Oh M, Komatsu S. 2015. Characterization of proteins in soybean roots under flooding and drought stresses. J. Proteomics 114: 161-181.

Pang XM, Zhang ZY, Wen XP, Ban Y, Moriguchi T. 2007. Polyamines, all-purpose players in response to environment stresses in plants. Plant Stress 1: 173-188.

Pulla RK, Kim YJ, Parvin S, Shim JS, Lee JH, Kim YJ, et al. 2009. Isolation of S-adenosyl-L-methionine synthetase gene from Panax ginseng C.A. meyer and analysis of its response to abiotic stresses. Physiol. Mol. Biol. Plants 15: 267-275.

Qi YC, Wang FF, Zhang H, Liu WQ. 2010. Overexpression of suadea salsa S-adenosylmethionine synthetase gene promotes salt tolerance in transgenic tobacco. Acta 
Physiol. Plant. 32: 263-269.

Roeder S, Dreschler K, Wirtz M, Cristescu SM, van Harren FJ, Hell R, et al. 2009. SAM levels, gene expression of SAM synthetase, methionine synthase and ACC oxidase, and ethylene emission from N. suaveolens flowers. Plant Mol. Biol. 70: 535-546.

Roy BN, Basu RN, Bose TK. 1972. Interaction of auxins with growth-retarding, -inhibiting and ethylene-producing chemicals in rooting of cuttings. Plant Cell Physiol. 13: 1123-1127.

Saitou N, Nei M. 1987. The neighbor-joining method: A new method for reconstructing phylogenetic trees. Mol. Biol. Evol. 4: 406-425.

Shen B, Li C, Tarczynski MC. 2002. High free-methionine and decreased lignin content result from a mutation in the Arabidopsis S-adenosyl-L-methionine synthetase 3 gene. Plant J. 29: 371-380.

Smirnova OA, Isaguliants MG, Hyvonen MT, Keinanen TA, Tunitskaya VL, Vepsalainen J, et al. 2012. Chemically induced oxidative stress increases polyamine levels by activating the transcription of ornithine decarboxylase and spermidine/spermine-N1-acetyltransferase in human hepatoma HUH7 cells. Biochimie 94: 1876-1883.

Uváčková L, Takáč T, Boehm N, Obert B, Samaj J. 2012. Proteomic and biochemical analysis of maize anthers after cold pretreatment and induction of androgenesis reveals an important role of anti-oxidative enzymes. J. Proteomics 75: 1886-1894.

Van Breusegem F, Dekeyser R, Gielen J, Van Montagu M, Caplan A. 1994. Characterization of a S-adenosylmethionine synthetase gene in rice. Plant Physiol. 105: 1463-1464.

Wawrzynska A, Moniuszko G, Sirko A. 2015. Links between ethylene and sulfur nutrition-a regulatory interplay or just metabolite association? Front. Plant Sci. 6: 1053.

Yang PF, Li XJ, Liang Y, Jing YX, Shen SH, Kuang TY. 2006. Proteomic analysis of the response of Liangyoupeijiu (super high-yield hybrid rice) seedlings to cold stress. J. Integr. Plant Biol. 48: 945-951.

Yi LF, Wang P, Zhou XH, Liu CW. 2009. cDNA cloning and bioinformatic analysis of SAMS gene from Porphyra yezoensis. China Biotechnol. 29: 43-49. 